\title{
Vaccination - un problème non résolu et de brûlante actualité
}

\author{
Interview avec Mme le Professeur Claire-Anne Siegrist, 20 octobre 2004
}

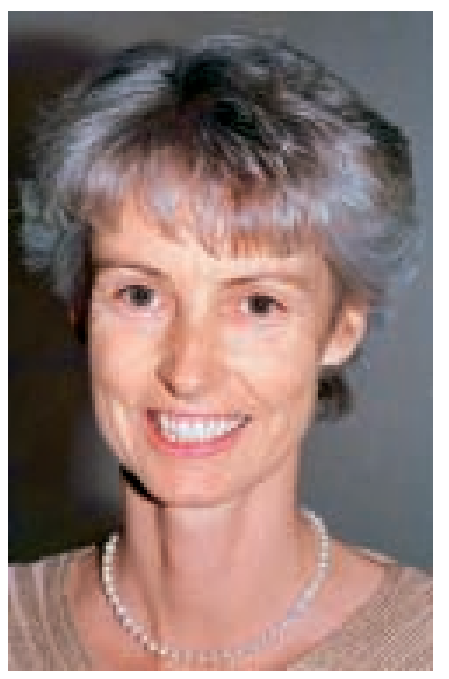

La population suisse est-elle dans I'ensemble bien vaccinée?

La population est dans l'ensemble bien vaccinée, même s'il existe des insuffisances bien documentées. Les enfants sont bien mieux protégés que les adultes, qui ont tendance à oublier les vaccins jusqu'à la veille d'un départ en voyage! Certains vaccins sont très demandés - ceux qui protègent contre des maladies qui font peur - alors que d'autres, comme celui contre la rougeole, demeurent un peu moins bien acceptés parce que l'on a tendance à banaliser l'importance des maladies contre lesquelles ils protègent.

Comment situer la position de la Suisse par rapport aux pays voisins?

On vaccine moins bien en Suisse - et de loin qu'aux Etats-Unis, dans le Nord de l'Europe ou même dans toute l'Europe de l'Est, soit dans tous les pays où les programmes de vaccinations sont gérés directement par la santé publique, que les vaccins soient totalement facultatifs comme en Angleterre ou en Finlande ou plus ou moins obligatoires comme à l'Est. Et on vaccine «aussi mal» qu'en France, en Allemagne ou en Italie ... des pays qui sont par exemple avec nous dans le peloton de queue pour la vaccination contre la rougeole, au point de compromettre son élimination de la Région Européenne!

\section{Que signifie concrètement cette expression "peloton de queue»?}

Cela signifie que le pourcentage d'enfants vaccinés (environ $85 \%$ ) reste insuffisant pour interrompre la circulation du virus, qui nécessiterait un taux de vaccination de $93 \%$. S'il n'y a plus de rougeole en Finlande, ce n'est pas parce qu'ils ont de meilleurs vaccins, puisque les mêmes vaccins sont utilisés par tout le monde. La différence réside seulement dans le niveau de couverture atteint - et donc dans l'impact qu'a la vaccination sur la transmission des maladies contagieuses. On peut ainsi établir la carte de la Région Européenne et voir que le risque de rougeole dans chaque pays est inversement proportionnel à la couverture vaccinale. Par contraste, tous les pays vaccinent à peu près au même niveau contre le tétanos ou la polio, qui font peur parce qu'elles touchent le système neurologique...
Quel standard devrions-nous atteindre en matière de vaccination?

En terme de prévention des maladies, les objectifs des programmes de vaccination sont clairement définis pour chaque maladie. Ainsi, par exemple, l'objectif d'éradiquer la diphtérie et le tétanos néonatal a été atteint en Suisse, même s'il y a encore quelques cas de tétanos chez des personnes non vaccinées, le plus souvent des personnes âgées. Idem pour la polio, dont le dernier cas remonte à 1982. Par contre, les objectifs de voir disparaître la rubéole chez les femmes enceintes et diminuer suffisamment l'incidence de la rougeole, des oreillons, de la coqueluche ou de l'hépatite B pour en éviter les complications graves ne sont pas encore atteints chez nous. Ces objectifs ne sont pas des «objectifs suisses», mais des objectifs spécifiques de santé publique visant à la prévention de maladies potentiellement graves ou mortelles. On connaît la couverture vaccinale à atteindre pour les voir disparaître. Par exemple, 93\% des enfants doivent être vaccinés pour contrôler la rougeole. On peut ainsi comparer facilement la couverture vaccinale qu'on obtient dans une région, un canton ou un pays et se positionner ensuite par rapport à l'objectif à atteindre. C'est ce qui me fait dire que la Suisse est dans le peloton de queue!

Combien de vaccins devraient-ils être appliqués pour atteindre ces objectifs prioritaires?

En Suisse, des objectifs de santé publique ont été précisés pour une dizaine de maladies (diphtérie, tétanos, coqueluche, poliomyélite, infections invasives à $H$. influenzae du groupe b, rougeole, rubéole, oreillons, hépatite $\mathrm{B}$, grippe chez les personnes âgées) contre lesquelles il existe des vaccins sûrs et efficaces. Ces vaccins sont considérés comme indispensables et font donc partie de ce qu'on appelle le plan de vaccination de base, ou «de routine». Ces vaccins ont un bénéfice évident pour la santé individuelle et pour la santé publique. D'autres vaccins ont un bénéfice plus marqué chez les patients faisant partie de groupes à risques spécifiques élevés.

Comment définir ces «groupes à risque»?

La surveillance des infections, par les médecins et les laboratoires, permet de recenser les infec- 
tions provoquées par un pathogène, par exemple par les hépatites ou les pneumocoques. On peut ainsi déterminer quelles personnes elles touchent, quelles classes d'âge sont concernées et cela permet - parfois - d'identifier des facteurs de risque. Cela peut être un risque accru d'exposition (profession, voyage, entourage d'un patient infecté, etc.) et/ou un risque accru de complications sévères. Ce risque peut ensuite être quantifié plus précisément par des études comparant la prévalence des différents facteurs de risques parmi les patients. Par exemple, l'utilisation de drogues intraveineuses et les pratiques sexuelles sont des facteurs de risque pour l'hépatite B ... même si 30-40\% des infections surviennent chez de jeunes adultes sans facteur de risque identifié.

\section{Existe-t-il un groupe à risque insuffisamment} vacciné par rapport au risque auquel il est exposé? Oui. Paradoxalement, la difficulté est précisément d'atteindre ces groupes à risque. On s'en aperçoit par exemple pour la grippe. Même si la situation s'améliore progressivement, seulement une partie des personnes âgées ou malades qui devraient bénéficier de cette vaccination est réellement vaccinée chaque année. Il y a malheureusement de nombreux patients à risque qui «passent entre les gouttes», qu'ils soient des nourrissons de mères avec une hépatite $B$ ou des malades pris en charge pour une pathologie X. Nous avons une médecine libérale qui implique que chacune et chacun bénéficie d'un traitement chez un ou parfois plusieurs médecins, sans registre centralisé. L'importance accordée à la thérapeutique, aux mesures immédiatement nécessaires, fait encore trop souvent passer la prévention à l'arrière-plan. Il faudrait que chaque médecin soit en mesure d'informer encore mieux les patients quant à la nécessité de mesures préventives. C'est un problème général, qui se double pour les vaccinations d'un problème de formation médicale continue: la vaccinologie est une science relativement jeune à laquelle les médecins n'ont été que peu exposés au cours de leur formation.

Avant de parler des critiques émises à l'égard des vaccins, je souhaiterais brièvement rappeler leur immense succès dans I'histoire de la médecine. Le succès des vaccinations est effectivement flagrant. L'un des plus grands succès, l'éradication de la variole, a été obtenu par des programmes de vaccination intensifs permettant d'atteindre progressivement une couverture vaccinale d'au moins $80 \%$ dans chacun des pays du monde. Au fur et à mesure que chaque pays atteignait ce seuil de $80 \%$, les efforts se sont concentrés sur l'identification des cas, leur isolement, la vaccination de l'entourage ... jusqu'à l'éradication certifiée depuis le 8 mai 1980, et donc l'arrêt de la vaccination.

Un deuxième succès majeur est la poliomyélite. En 2004, il n'y a plus que 6 pays (Inde, Pakistan, Afghanistan, Nigeria, Niger, Egypte) dans lesquels la poliomyélite est encore endémique. Son éradication dépend maintenant essentiellement des événements au Nigeria, le seul pays où les cas de paralysie ont augmenté (28 en 2002, 796 en 2004) au lieu de diminuer. Pourquoi? Parce que les campagnes de vaccination de l'Organisation mondiale de la santé (OMS) ont été interrompues par les allégations de certains chefs religieux affirmant que le programme de vaccination de l'OMS faisait partie d'une conspiration américaine visant à réduire la population musulmane en stérilisant les femmes ou en transmettant le SIDA aux enfants. Des études ont du être effectuées pour leur démontrer que les vaccins contre la polio n'étaient contaminés ni par des anti-contraceptifs ni par le VIH. Même cela n'a pas suffi à effacer les craintes, comme souvent lorsqu'elles sont irrationnelles, si bien qu'il a fallu obtenir pour le Nigeria des vaccins fabriqués dans un pays musulman! Malheureusement, l'interruption des campagnes de vaccination a provoqué le rebond de la poliomyélite au Nigeria et l'exportation vers six pays voisins qui avaient déjà éliminé la poliomyélite de leur territoire. Les efforts ont repris et l'objectif de l'OMS reste de pouvoir éliminer la poliomyélite en 2005. Mais il faut des efforts considérables et des campagnes massives qui «arrosent» les régions concernées de virus vaccinal, pour que les lignées de virus sauvages s'éteignent et disparaissent. L'éradication de la poliomyélite a donc été mise en danger par une polémique irrationnelle. Son avenir se jouera sans doute en 2005, dans quelques pays du monde. Si ce but n'était pas atteint dès 2005, l'éradication pourrait devenir très difficile. Maintenir les efforts à leur niveau actuel serait en effet plus onéreux que ce que les puissants de ce monde sont prêts à payer ...

Un troisième succès majeur de la vaccination est la disparition de la diphtérie, puisqu'elle a disparu de tous les pays ayant introduit et maintenu une couverture vaccinale suffisante. Mais la diphtérie reste endémique dans de nombreux pays, y compris dans des pays à quelques heures d'avion (Algérie, Egypte, Albanie et les pays de l'ex-URSS). Et elle ne demande qu'à revenir. La preuve: en 1995, une épidémie de 50000 cas de diphtérie a frappé plusieurs républiques de l'ex-URSS à cause d'une diminution de la couver- 
ture vaccinale des petits enfants. Il a fallu des campagnes de vaccination nationales des enfants et des adultes pour y mettre fin.

En Suisse, nous assistons apparemment à un scepticisme grandissant à l'égard des vaccins. Ces derniers seraient-ils victimes de leur propre succès? Ne peut-on pas, d'une certaine manière, comprendre les parents qui ne voient plus I'utilité de vacciner contre des maladies qu'ils ne connaissent plus et qui ne font plus peur?

Bien sûr, c'est la rançon de la gloire! Pourquoi se protéger de ce qui ne fait pas peur? Ce phénomène est aussi le résultat d'une évolution vers une société de plus en plus individualiste, où la notion de solidarité est passée de mode. Certaines mères ont la franchise de dire: «L'idéal pour moi serait que tous les enfants soient vaccinés - sauf le mien. Comme cela, mon enfant n'encourrait ni le risque d'effets secondaires, ni celui de la maladie.»

\section{Mais c'est une attitude cynique!}

Sans doute. Mais elle n'est pas spécifique à la vaccination! Regardez les téléphones portables: tout le monde en veut, mais personne ne veut une antenne sur son immeuble ou dans son quartier. Chacun prend l'autoroute, mais personne n'accepte une bretelle devant son balcon. A cet individualisme croissant de notre société, qui me préoccupe, s'ajoute un déficit d'information, l'oubli des maladies qu'on ne voit plus, une certaine méfiance face aux autorités en général et aux autorités de santé en particulier ... et cela suffit à faire réapparaître des questions auxquelles on croyait avoir répondu en démontrant les succès de la vaccination.

Ne pourrait-on pas, tout aussi cyniquement, souhaiter que certaines maladies connaissent un renouveau pour que les patients soient à nouveau plus enclins à se faire vacciner! C'est malheureusement ce qui se passe! On n'a jamais autant vacciné contre la rougeole qu'en 2003, pendant une épidémie de rougeole pendant laquelle près de 700 cas ont été déclarés en Suisse - sans doute la pointe d'un iceberg cinq fois plus important. Les parents se sont précipités chez les médecins, stupéfaits d'apprendre que la rougeole existait encore en Suisse! Ces 700 cas ont donc fait plus que de longs discours ... Pourtant, je ne peux m'empêcher de penser aux 95 enfants ayant souffert de complications parfois graves, puisqu'il y a eu quatre encéphalites.
La critique des programmes de vaccination puise donc ses racines dans la psychologie humaine.

Oui, même s'il faut aussi regarder ce qui n'a pas été fait correctement par le corps médical. Je pense que les médecins en général - et les autorités de santé en particulier - ont trop tardé à prendre la juste mesure des changements intervenus dans les mentalités et à faire bénéficier la population des informations nécessaires. Je parle bien sûr spécifiquement des vaccins, mais ma remarque concerne bien d'autres domaines de la médecine où il a fallu bien du temps pour passer d'une relation paternaliste au partenariat de plus en plus souvent réclamé par les patients confrontés à des choix pour leur santé. Malheureusement, la qualité et la quantité des informations mises à disposition des médecins et des patients est restée très longtemps insuffisante. Elle l'est d'ailleurs encore, et cela contribue à créer des brèches dans lesquelles vient par exemple s'engouffrer la brochure distribuée par la Fondation pour la protection des consommateurs.

\section{Parlons alors de cette fameuse brochure intitulée} «Guide pour une vaccination différenciée»:

ses auteurs se croient-ils appelés à partir en guerre contre les vaccins?

Apparemment. Les naturopathes évoluent dans un système de valeurs avec des concepts différents de ceux de la médecine fondée sur les preuves. Cette idéologie a toujours existé et est digne de respect. Elle postule que «la Nature est bonne» et donc que les maladies sont utiles au développement de l'enfant, voire qu'elles sont des épreuves que la vie nous envoie pour nous renforcer, puisque le décès lui-même fait partie de la nature et doit pouvoir être accepté. La majorité des naturopathes reconnaissent sans difficulté que tout comme certaines pathologies dépassent le cadre des médecines alternatives et nécessitent des traitements allopathiques, la prévention vaccinale n'est pas en opposition à une médecine naturelle. Pour d'autres, une très petite minorité, la règle de laisser faire la Nature est absolue - donc la vaccination est une ingérence dans l'évolution naturelle. Ceux-ci estiment qu'en prévenant une maladie par une vaccination, on prive l'enfant d'occasions de développement et qu'on l'expose à des perturbations responsables ultérieurement de maladies chroniques aussi diverses que les allergies, les maladies auto-immunes ou le cancer. Entre les deux, certains naturopathes tentent de faire la part des choses en proposant de vacciner de façon «sélective», en choisissant certains vaccins, en les retardant, etc. 
Aucun parent ne vaccine son enfant sans se poser des questions au préalable. La plupart s'en ouvrent à leur pédiatre ou leur médecin. Malheureusement, nous, médecins, ne répondons pas toujours de façon optimale aux questions et attentes des parents. Mais comment faire alors que l'on n'a bénéficié que d'une seule heure de cours sur les vaccins pendant toute sa formation médicale? Dans le passé, le bénéfice des vaccinations paraissait tellement évident qu'il n'y avait pas besoin d'en savoir plus. Il fallait juste apprendre la liste des vaccins et «2, 4, 6 mois» par cœur pour passer l'examen avec succès! Dans l'intervalle, la vaccinologie est devenue une science à part entière, exigeant des connaissances en infectiologie, en immunologie moléculaire et cellulaire, en épidémiologie et en santé publique. Il y a donc un déficit de formation et d'information des autorités de santé vers les médecins, puis des médecins vers les parents. Certains parents plus inquiets ou hésitants cherchent alors d'autres sources d'information, et tombent parfois sur la brochure distribuée par la Fondation pour la protection des consommateurs. Cette Fondation ayant jusqu'ici une réputation de sérieux, ils pensent trouver dans ce guide des informations équilibrées sur les avantages et les risques des vaccinations. Malheureusement, il n'en est rien.

Les auteurs de cette brochure auraient-ils mal apprécié les évidences scientifiques?

En réalité, les trois auteurs de cette brochure mettent sur pied d'égalité des concepts idéologiques de naturopathie, comme par exemple l'influence des maladies sur le développement de l'enfant, et les évidences scientifiques rassemblée par la médecine fondée sur les preuves. Le problème, c'est que les référentiels sont totalement différents. Cette brochure devrait être conçue pour aider les gens à prendre une décision individuelle. En fait, les auteurs y transmettent leur idéologie personnelle en la présentant comme une évidence. En choisissant très sélectivement les exemples qu'ils citent, ils démolissent sciemment les vaccins, les programmes de vaccination, les autorités de santé qui les recommandent et les médecins qui les appliquent. Il ne s'agit pas d'erreurs d'appréciation. On peut ouvrir cette brochure à n'importe quelle page et dresser une liste des aberrations, en découvrant par exemple la façon dont les citations ont parfois été modifiées pour faire croire que la maladie n'est pas si grave, que le vaccin ne marche pas si bien ou qu'il est beaucoup plus dangereux que ce que les autorités veulent bien faire croire.
Expliquez-nous un peu plus concrètement ce que vous reprochez à cette brochure.

Le principal problème est que sous prétexte d'informer objectivement, cette brochure distille le doute et la méfiance face aux médecins: si un médecin conseille de vacciner en suivant les recommandations de l'Office fédéral de la santé publique (OFSP), ce qui est appelé une «variante maximale», cela signifie implicitement qu'il est incompétent, mal informé ou manipulé par les autorités de santé et/ou l'industrie. Mettre en péril la confiance des patients en leur médecin est un jeu dangereux, et on peut se demander à qui il profite. Deuxièmement, suivre les conseils de cette brochure fait aboutir à des impasses. Par exemple il est affirmé qu'il ne faut surtout pas vacciner un enfant né prématurément (!), mais il est par contre écrit un peu plus loin que le vaccin contre la méningite pourrait être considéré pour les enfants nés prématurément qui sont à risques plus élevés. Comment voulez-vous vous y retrouver? Cela génère le doute, donc l'abstention ...

Le plus grave est que cette brochure passerait pratiquement inaperçue si elle était publiée à compte d'auteurs. Mais sa distribution par l'intermédiaire d'une Fondation qui a pour mission de protéger les consommateurs lui donne une pseudo-crédibilité, une pseudo-objectivité qu'elle est loin d'avoir. Que cette brochure soit sponsorisée par une Fondation qui a certainement à cœur d'avoir la meilleure crédibilité possible m'interpelle ...

\section{Pour le cas où surgiraient des conséquences} touchant la morbidité ou la mortalité infantiles, les auteurs de la brochure ou la Fédération suisse des consommateurs ont-ils une responsabilité sur le plan juridique?

Je ne peux pas répondre sur le plan strictement juridique. Les auteurs n'hésitent d'ailleurs pas à se couvrir en écrivant en première page de leur brochure que chacun est responsable de ses décisions et qu'aucune poursuite juridique ne peut leur être intentée! Mais au niveau moral, leur responsabilité me paraît très clairement engagée ... de même que j'accepte d'engager ma propre responsabilité médicale chaque fois que je conseille une vaccination en expliquant pourquoi.

Que pouvons-nous faire aujourd'hui pour endiguer la propagation de cette désinformation?

De très nombreux médecins, y compris à la Commission suisse des vaccinations, attendaient de l'OFSP - qui est directement attaqué à chaque 
page de cette brochure - qu'il en dénonce les aberrations et soutienne ainsi les médecins chargés d'appliquer ses recommandations. Le silence persistant des autorités a décidé un groupe de pédiatres spécialistes en maladies infectieuses à consacrer les centaines d'heures de travail nécessaires pour rassembler les réponses aux principales questions suscitées par cette brochure (voir article en annexe). Nous espérons trouver les moyens de rendre ce travail accessible aux médecins et de faire savoir publiquement que cette brochure n'est pas un guide objectif pour une vaccination différenciée.

Cette démarche correspond à ce qui me semble le plus urgent: pallier au déficit actuel d'information dans le domaine de la prévention en général, et des vaccinations en particulier. Prendre conscience de la complexité - croissante - du domaine de la vaccination, qui nécessite des connaissances en épidémiologie, en immunologie, en infectiologie, en pédiatrie et en santé publique, pour l'aborder de façon correcte. Se donner plus de moyens pour procéder au détail des analyses nécessaires. Prendre conscience de la demande d'information et transmettre cette dernière de façon encore plus complète et différenciée. C'est dans ce but qu'a été créé un réseau d'experts destiné spécifiquement aux médecins, avec le soutien de l'OFSP, de la Société Suisse de Pédiatrie et de la Société Suisse d'Infectiologie. Ce réseau (InfoVac, www.infovac.ch) distribue des bulletins d'information en prise directe sur l'actualité et permet de recevoir en 24-48 h une réponse personnalisée à toute question concernant une vaccination. Le succès de cette initiative (plus de 2600 médecins déjà abonnés, plus de 2000 questions reçues chaque année) a confirmé son utilité, voire sa nécessité.

Dans le même objectif, l'OFSP a demandé au Département fédéral de l'intérieur de promouvoir la Commission suisse des vaccinations au titre de Commission fédérale pour les vaccinations, afin de lui donner l'indépendance académique nécessaire. Cette commission est composée de membres dont les compétences couvrent tous les aspects de la vaccinologie et dont les dossiers ont été analysés par les juristes et estimés ne pas contenir de conflits d'intérêt susceptibles de limiter leur objectivité. Le mandat de cette commission est d'abord de conseiller nos autorités pour tout ce qui concerne les programmes de vaccination. Mais c'est aussi d'en exposer les évidences et de mieux communiquer les considérations à la base des recommandations. Nous avons donc commencé par établir des priorités et définir nos méthodes de travail. Par exemple, chaque nouveau vaccin sera analysé selon un cadre analytique détaillé. Le dossier qui en résul- tera sera rendu public, de façon à ce que chacune de nos recommandations soit dûment étayée. Je pense que cette démarche répond à l'attente de nos autorités, des médecins et du public, et qu'elle facilitera des choix et décisions reposant sur des évidences scientifiques.

Votre commission sera-t-elle amenée à donner des conseils politiques? Faudrait-il par exemple remettre la vaccination entre les mains des médecins d'école plutôt que de la laisser aux pédiatres et médecins de premiers recours? Cela n'est pas si simple! Les petits enfants ne vont pas encore à l'école, et il y a autant de systèmes de santé scolaire que de cantons. Si certains cantons sont très efficaces en matière de vaccination, d'autres sont moins actifs. La couverture de la vaccination contre l'hépatite $\mathrm{B}$, par exemple, varie entre 7 et $84 \%$ selon les cantons. Mais effectivement le rôle de l'école apparaît comme essentiel, surtout pour les vaccins destinés aux grands enfants ou adolescents. Il y a donc toute une réflexion à mettre en route sur les mesures de prévention de demain. Nous allons avoir à disposition des vaccins de plus en plus sophistiqués contre un nombre toujours plus grand de maladies. Il y aura des vaccins contre les maladies contagieuses mais aussi contre certaines maladies chroniques. Et des vaccins thérapeutiques à offrir aux patients malheureusement déjà infectés, pour aider leur système immunitaire à enrayer l'infection. Notre société devra se positionner clairement par rapport à toutes ces questions de prévention primaire et secondaire. Il y encore beaucoup à faire en Suisse pour donner à la prévention ses lettres de noblesse et pour obtenir les budgets nécessaires. Nous sommes encore extrêmement limités dans nos moyens d'informations et dans la prise en charge de la prévention par les assurances. En 2005, la prévention reste le parent pauvre d'une médecine de pointe de plus en plus technique limitée à un petit nombre de bénéficiaires. J'ignore quel sera le poids de notre Commission dans ce débat. C'est à plus long terme que nous devrons aborder ces questions.

\section{Qui la Commission conseille-t-elle?}

La Commission conseille l'OFSP et le Département fédéral de l'intérieur (DFI). En tant que commission fédérale, notre indépendance de communication est maintenant garantie. Une de nos premières décisions a été de créer un groupe de communication pour déterminer ce que nous voulons communiquer, à qui, par quels canaux, avec quelle urgence et par quels moyens. Nous allons fonctionner comme toutes les commissions fédérales, c'est-à-dire en système de milice 
avec des groupes de travail constitués autour d'une question spécifique et chargés de préparer les documents nécessaires aux débats en séance plénière, qu'il s'agisse d'établir des recommandations pour de nouveaux vaccins ou de prendre position par rapport à des thèmes prioritaires. Le travail ne manque pas.

Est-ce que les restrictions budgétaires que subit I'OFSP touchent directement votre commission? Oui. D'une part parce que notre Commission n'a pas de budget spécifique et que nous dépendons de l'OFSP, par exemple pour faire une expertise ou réaliser un mandat. D'autre part parce que les meilleures des recommandations restent inopérantes si elles ne sont pas communiquées aux médecins et au public par des campagnes d'information. Si le budget de la santé publique est encore réduit, la Suisse restera dans le peloton de queue en matière de prévention.

Interview: M. Trutmann et P. Bonfils

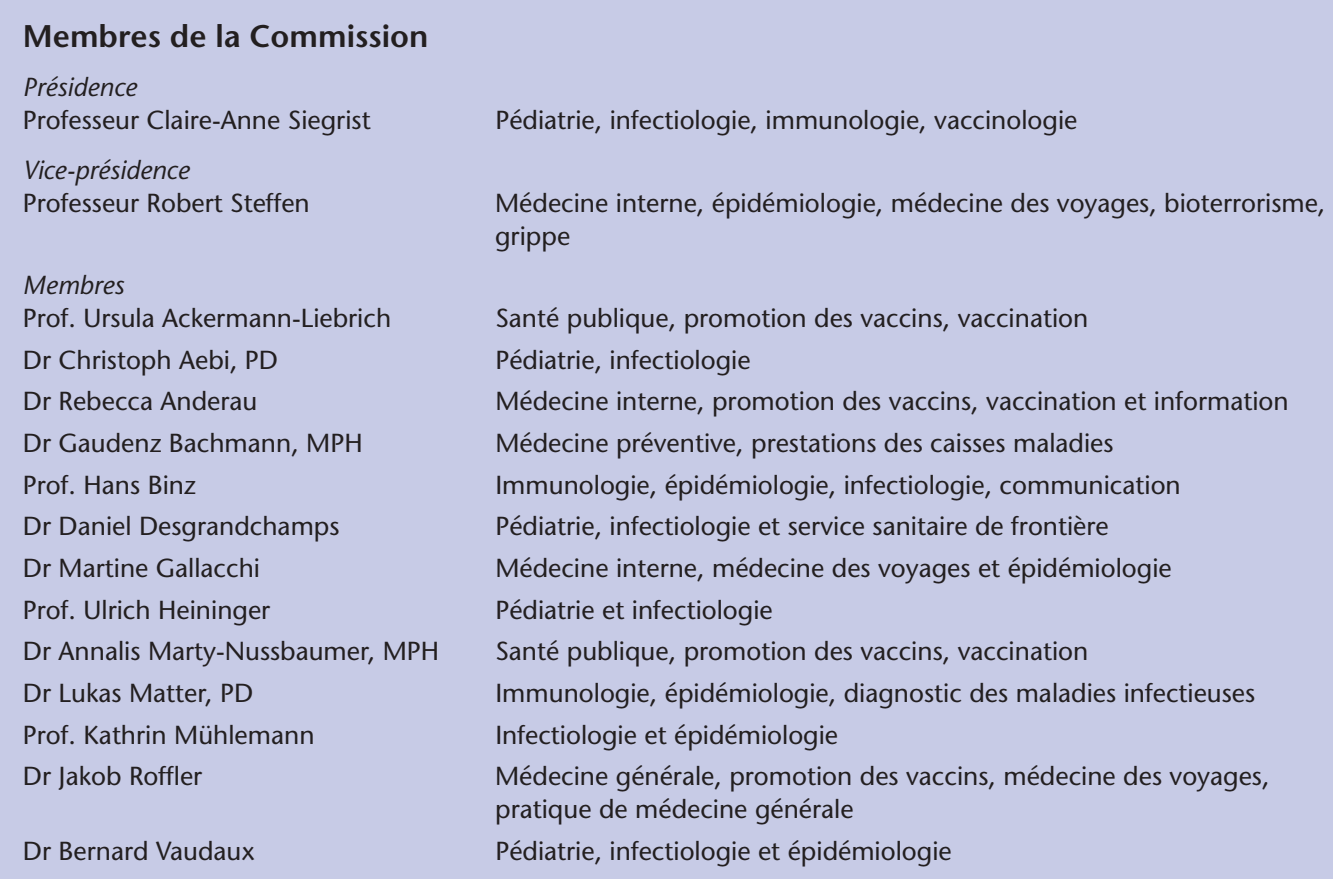

\section{Critères d'évaluation de nouveaux vaccins, pour l'élaboration de recommandations nationales en Suisse.}

Question 1: Le fardeau de la maladie justifie-t-il la mise en œuvre d'une recommandation de vaccination?

Question 2: $\quad$ Les caractéristiques du vaccin vont-elles permettre la mise en œuvre d'une recommandation de vaccination efficace?

Question 3: Quelles sont les stratégies permettant d'atteindre le but recherché par une recommandation de vaccination?

Question 4: Les indices coût-efficacité des stratégies sont-ils acceptables et comparables à d'autres interventions de santé?

Question 5: Un niveau élevé de demande ou d'acceptation existe-t-il pour la recommandation de vaccination?

Question 6: L'application de la recommandation de vaccination est-elle réalisable?

Question 7: Les différents aspects de la recommandation sont-ils évaluables?

Question 8: $\quad$ Existe-t-il d'importantes questions ouvertes qui conditionnent la mise en œuvre de la recommandation?

Question 9: La recommandation est-elle équitable en termes d'accessibilité du vaccin pour l'ensemble des groupes cibles?

Question 10: Existe-t-il des problèmes juridiques qui conditionnent la mise en œuvre e la recommandation?

Question 11: La recommandation planifiée est-elle conforme avec celles prévues ou planifiées dans d'autres endroits? 